\title{
An Optimized Two-Purpose Peer-to-Peer VANET to Disseminate Traffic Information and Reduce Travel Time
}

\author{
Ali Asghar Valibak, Nik Mohammad Balouchzahi, Kaamran Raahemifar, Mahmood Fathy, and Ahmad \\ Akbari
}

\begin{abstract}
In this paper, we propose a two purpose structure that can do vehicle navigating and packet routing simultaneously in an efficient way. The algorithm suggests the shortest travel time path to the drivers and finds reliable routes for traffic information messages. In order to reduce traveling time, vehicles are directed to the paths with lower car congestion. Because such paths have a higher risk of radio coverage partitioning, it is more desired to disseminate traffic information packets from routes that have more probability of radio connectivity, to increase successful ratio on delivering messages. For this purposes, it is necessary to assess vehicles distribution on city roads periodically to evaluate real-time car congestion and radio connectivity of roads. The peer-to-peer layer is constructed in a fast and simple way, without any overhead or extra message exchanging between nodes. The system can optimally save the limited bandwidth of vehicular network as well as reducing the travel time of vehicles. Due to completely cooperative characteristic of this system, there is no need to install any infrastructure at road network.
\end{abstract}

Index Terms-Two-purpose, VANET, ITS, TIS, navigation, routing.

\section{INTRODUCTION}

In recent years, due to increasing of urban car traffic all around the world, Traffic Information Systems (TIS) that collect and distribute traffic conditions were being interested by researchers. In traditional traffic information systems, the data are collected by some installed sensors like cameras and are sent to a management and control center by means of transmission network. After processing the data, the real-time traffic conditions are shown on road sign screens or are sent to vehicles navigation systems to be used by drivers. The main problem of this system is their need to expensive infrastructure and their dependency on management and control centers. Furthermore, their time delay is in range of few minutes, which might not be suitable for some traffic applications.

Beside the introduction of Vehicular Ad-hoc NETworks (VANETs), Cooperative Traffic Information Systems (CTIS) as a new generation of TISs began to be implemented in Intelligent Transportion Systems (ITS). In CTISs, moving

Manuscript received September 10, 2014; revised November 20, 2014. Ali Asghar Valibak, Nik Mohammad Balouchzahi, Mahmood Fathy, Ahmad Akbari are with the Department of Computer Engineering, Iran University of Science and Technology, Tehran, Iran (e-mail: valibak@gmail.com, balouchzahi@iust.ac.ir, mahfathy@iust.ac.ir, akbari@iust.ac.ir).

Kaamran Raahemifar is with the Department of Electrical and Computer Engineering, Ryerson University, Toronto, Ontario, Canada (e-mail kraahemi@ee.ryerson.ca). vehicles act as network nodes and all traffic data are being collected, distributed and mostly edited by themselves. They can use VANETs or installed cellular networks or both.

In this paper, we propose a CTIS based on a Peer-to-Peer (P2P) VANET that aimed to reduce travel time in cities for moving vehicles. This is an optimized and advanced of our previous works [1], [2]. For implementing this system, there is no need to install any infrastructure at streets. This algorithm not only finds the best path for driving, but also tries to find the best route for sending packets, in order to optimize using the limited bandwidth of VANETs. To overcome the challenge of limited bandwidth of VANETs, data caching in nodes is being used for a specified and limited period of time.

In this article, the next part will review the related works in field of Cooperative Traffic Information Systems. In the third part, we will introduce the proposed approach and describe how it acts. In chapter four, we discuss system simulation results, and its performance in different conditions will be analyzed. Conclusions and future works will be stated lastly.

\section{RELATED WORK}

In some CTISs, vehicles do data collecting, but this information must be sent to a management and control center to be analyzed. [3]-[5] are examples of these systems, using cellular networks [6]. At the other cooperative systems, central management and control part has been replaced by a multilayer architecture where the analysis of traffic data is done inside each moving node. Based on peer-to-peer layer formation and usage of cellular or vehicular networks, these structures have been divided into four different categories [7]:

- Single-tier VANETs

- Single-tier peer-to-Peer VANETs

- Single-tier peer-to-peer infrastructure-based systems

- Two-tier peer-to-peer systems

Single-tier traffic information systems are those that completely use either pre-installed infrastructure-based networks (such as $\mathrm{GSM}^{1}$, $\mathrm{UMTS}^{2}, \mathrm{LTE}^{3}, \mathrm{WIMAX}^{4}$ ) or infrastructure-free networks (like VANETs with or without Road Side Units (RSU)). They me have one layer or two different layers. In case of two layers architecture, the overlay is a peer-to-peer layer. Two-tier systems; that are

\footnotetext{
${ }^{1}$ Global System for Mobile

${ }^{2}$ Universal Mobile Telecommunications System

${ }^{3}$ Long-Term Evolution

${ }^{4}$ Worldwide Interoperability for Microwave Access
} 
called Hybrid TISs as well, are those systems that use both infrastructure-based and infrastructure-free technologies in two different layers. In this case, underlay layer shall be a VANET and the overlay is a peer-to-peer cellular network.

Single-tier VANET TISs such as [8]-[11] are in fact a traditional VANET and is the only single tier system that does not be benefited from peer-to-peer layer. The main drawbacks of these networks are partitioning of the radio coverage, and broadcasting storm in searching traffic information. In these systems it is important to prevent flooding, collision and dropping of traffic data packets and avoiding saturation in limited bandwidth of wireless network when data traffic is at the peak.

Single-tier P2P VANET TISs like [12]-[15] use the same vehicular networks to exchange traffic information between nodes as previous one. The only difference is that traffic information is only distributed in the paths that are located between the source and destination nodes, instead of broadcasting in the whole network. These systems are more tolerated against rapid saturation of bandwidth and the flooding of traffic information in whole network. The great challenge of these systems is radio coverage discontinuity of VANET that act as a single platform for telecommunication between nodes. Therefore, packets of traffic information in this structure may get lost at high rates.

Single-tier peer-to-peer TISs such as [16], [17] are based on cellular networks in both underlay and peer-to-peer layer. These systems may use one of GSM, 3G or 4G technologies of cellular mobiles or may use WIMAX for telecommunicating between nodes. Using this type of infrastructure-based networks, traffic messages are received with higher latency compared to the vehicular ad-hoc networks, and peer-to-peer layer will be formed slower in this structure, too. In contrast, this system will be safe from partitioning problem of VANETs.

To have advantages of low delay Inter-Vehicle Communications (IVC) and at the same time, using continuous radio coverage of cellular networks, two-tier structures like [18], [19] were introduced. These systems have VANET as a platform to disseminate information in underlay layer. The peer-to-peer layer uses cellular networks for telecommunication between peers on overlay. Due to the simultaneously use of two different networks, packet overheads on this structures are more than other architectures and peer-to-peer layer formation is more complex than single-tier structures.

Based on the above mentioned structures, some algorithms in context of cooperated traffic information system have been proposed. A group of these algorithms are presented with the aim of reducing congestion of vehicles on urban roads. The other group emphasizes on improving traffic information packets disseminating.

In this paper, the proposed method is based on the second structure which is a Single-tier Peer-to-Peer VANET Cooperative Traffic Information System. One of the key differences between proposed algorithm and previous related works mentioned earlier is that this Two-Purpose algorithm is covering both vehicle navigating and traffic information packet routing simultaneously.

\section{PROPOSED MeTHOD}

In this section a Single-tier Peer-to-Peer VANET System is presented that can do vehicle navigation and packet dissemination simultaneously. It is necessary that each vehicle has been equipped with a GPS, a digital map, an 802.11p transceiver, a CPU and a memory to store information. Digital map included roads and junctions identified by global unique IDs. Roads between two consecutive junctions are called road segments. Different directions of each road segment have two different IDs. For each road segment, the maximum speed limit is specified too. All the vehicles moving in a road segment are considered as a cluster.

In this system, all traffic information is stored locally in each moving node using three local tables and this information can be exchanged between nodes using four type of packets. Therefore, there is no management and control center to direct the system activity. All the participating vehicles are categorized into two different node types according to their role in the network:

- Super-Peer nodes; that make the overlay peer-to-peer layer and are interfaces between overlay and underlay,

- Normal nodes; that only participate in VANET underlay layer.

To exchange the traffic information between nodes, four types of packets are used: Normal packets are broadcasted in a road segment and exchange information between moving vehicles in that road segment. This packet can be sent by both normal and super-peer nodes. Super-peer packets are broadcasted at junctions just by super-peer nodes to exchange information between super-peers that move in different road segments. Lookup packets that can be published by each node when it needs to search for updated road segment travel time information. Finally, Reply packets that published when a node receives a Lookup packet from others and the receiver node has the updated travel time information for the requested road segments.

To store and cache needed information, each node uses three types of information tables: Travel-Time Table stores aggregated experienced time for each road segment that is passed by own or other nodes. By using this table each node can find the shortest path to the destination that takes the least time to pass. Neighboring Table stores the location of vehicles that are moving in the same road segment. Each node can use this table to do super-peer selection procedure and do the VANET connectivity calculations. Finally, VANET-Connectivity Table caches the probable radio connectivity of VANET for each road segment. By using this table, each node can find a route for lookup packets toward their destination that has the most probability of VANET connectivity.

To save limited bandwidth of VANET from useless transmissions, all Normal packets and Super-peer packets are sent periodically by an adaptive interval depending on the vehicle speed.

Since this algorithm uses different sources for packet routing and vehicle navigating, the planned path for vehicles and packets may be different. Navigating tries to find a short and rapid way to destination, and routing tries to find a radio 
connected path. The proposed algorithm tries to fulfill these two goals.

\section{A. Updating Tables Information}

There are two different sources for updating the local cached information. First one is that information that each node can obtain itself according to its experience during passing each road segment; and second one is that information that can be achieved from other nodes. Each node aggregates new information with cached ones and then replaces old data with the updated one by attaching a time stamp to each entry. Searching the data in the tables is done by using unique Segment ID or Node ID as the entry keys.

The methods of updating the stored tables are different. Travel-time table uses an aggregation method to update information. Because the traffic condition is not changing very soon, traffic information that obtained during a couple of minutes are helpful for navigating plans.

To update information of Travel Time table at each node, (1) is used to aggregate new and old data for each road segment entry:

$$
T_{\text {Agg }}=\alpha T_{\text {Old }}+\beta T_{\text {New }}
$$

where TAgg is aggregated travel time of related road segment that will be stored instead of old data, TOld is currently stored travel time of the road segment; TNew is the new received travel time of that road segment. Coefficients $\alpha$ and $\beta$ are defined as:

$$
\alpha=1 /(1+\Delta T) ; \beta=\Delta T /(1+\Delta T)
$$

where $\Delta T=$ TNew - TOld and $\alpha+\beta=1$. This formula controls the updating rate of traffic information in Travel-time table. It means that when the difference between time of last updated and time of receiving new data is too long, the new one has much more weight than the old one. Therefore, the table information will become freshen with the new data. On the other hand, when a node receives some similar data about a road segment in a short period of time, this formula try to control the effect of this new data by assigning a low weight to them by a small $\Delta \mathrm{T}$. These two advantages may prevent navigating part of the algorithm to plan according to very old or much fluctuating information. Some security benefits may be achieved from these controls too.

The updating of VANET-Connectivity table is different from the Travel-time table. Due to fast changes in cars location in the road network, the radio connectivity of road segments may change rapidly too. Therefore, it is necessary to replace cached data with the new received one without any aggregation. It means by receiving any new data about road segments radio connectivity, this table will be freshened soon and its data-changing rate is much more than Travel-time table.

The information of Neighboring table is updated in a fast way too. If a node does not receive new information about a saved neighbor during three consecutive broadcasting times, the host node removes this neighbor from its Neighboring table. If a node can receive new information from a node that shows the traveling road segment of them are the same, it adds this node as a neighbor in its Neighboring table, attaching a time stamp and the neighbor's position on lane. There are different sources to add and update neighbor information. If a node receives any packet directly from a node, it can easily check its moving road segment and compare with its own road segment. If there were the same, it adds that node as a neighbor. Receiving a Normal packet from other nodes and checking their Neighboring table is another source of obtaining neighbor nodes information. The main difference between the Neighboring table and the other two tables is that all of its entries are cleaned when the vehicle changes its moving road segment.

\section{B. Normal and Super-Peer Packets Broadcasting}

As a key problem of VANETs is the limited bandwidth for sending packets. Therefore, it is useful to control the rate of sending packets in such a way that prevent reaching the saturation thresholds. In our proposed system, there are two periodic broadcasting packets: Normal and Super-peer packets. To save the VANET bandwidth from sending useless data, the algorithm tries to transmit these two types of packets in adaptive intervals depending on the node's speed. It means that instead of transmitting these two packets in fixed intervals, each fast moving node can broadcast Normal and Super-peer packets more frequent than slow moving nodes using (3):

$$
I_{a}=I_{0}\left|40-v / V_{0}\right|
$$

where $I a$ is broadcasting interval for sending two consecutive Normal or Super-peer packets (Sec), I0 is a predefined interval for beacon sending in VANETs and is considered 0.1 Second, $\mathrm{v}$ is node's speed $(\mathrm{m} / \mathrm{s})$ and V0 is a reference speed $(\mathrm{m} / \mathrm{s})$ to control the change rate of the formula and is considered as 1 in this round of our project. Constant value of 40 has role of controlling minimum time interval in (3) according to this fact that the maximum valid speed of city roads is less than $40 \mathrm{~m} / \mathrm{s}(144 \mathrm{~km} / \mathrm{h})$. By using (3), fast moving cars can broadcast in shorter intervals than stopped or slow moving nodes.

\section{Super-Peer Selection}

The proposed system has two layers architecture. Some nodes shall act as cluster heads called super-peers to exchange traffic information between different clusters. They are also part of underlay VANET. Therefore, they can act as a bridge between the two layers.

Selecting super-peer nodes is an easy and fast process in this algorithm. They are the nodes that are the closest nodes in their clusters to the junctions. All the nodes that are moving in the same road segment make a cluster without considering the moving direction. Each node considers itself as a super-peer node when it enters a new road segment. Each vehicle then updates its local Neighboring table when it receives a packet. If it receives any packet directly from a node, it checks its sender road segment. If the sender's road segment was the same as receiver nodes, the sender ID will be added in the receiver node's Neighboring table. If the received packet was a Normal packet, the Neighboring table of the packet also is parsed and valid neighbor IDs will be entered in the receiver node's Neighboring table. The node then investigates its distance to the end junctions of the road 
segment with other neighbor nodes in its Neighboring table. If it was the closest node to one of the junctions of its moving road segment, it acts as a Super-peer node and will continue to have this role until receiving a packet from another node showing a neighbor vehicle in its cluster is closer to the junction than itself. Therefore, in this way of super-peer selection, there is no need to exchange any extra overhead or packet between nodes. It is also a fast way of selecting overlay peers that make peer-to-peer layer construction an easy and fast job. Changing the role from Normal peer to Super-peer has the same procedure too.

\section{Searching for Traffic Information}

One of the most important issues that have a high effect on a TIS system performance is the way of searching the traffic data from other participating nodes. In our algorithm, searching the traffic information is triggered whenever a moving node is reaching to a junction that has more than one path to select. At this time, the node renews the navigation from current location to the destination according to the information of Travel-time table. Then if the new planned path road segments have not updated information, a lookup packet has been released by host node containing out-of-date road segments list and updated information is requested. To be sure that this Lookup packet will travel via a reliable route, the algorithm uses VANET-Connectivity table to find a path from current location to the requested road segments that has the most radio connectivity probability and then emerges this planned route inside the Lookup packet. The packet route will be limited just to this planned route and only nodes that are moving in these road segments re-broadcast this Lookup packet and forward it for other nodes. Therefore, the number of Lookup packets that each node publishes is limited to the number of junctions during its trip path. In addition, the geographical path for each Lookup packet is defined by the originator node. Bounding the number and path of Lookup packets by the algorithm, help saving the bandwidth of underlay VANET. To prevent flood broadcasting problem, hop count limitation is implemented too. Although forwarding the Lookup packets is limited as mentioned, to reply the searching packets there is no need for answering node to be in the planned route.

\section{E. Estimating Probability of Routes Radio Connectivity}

In this algorithm, Lookup and Reply packets are sent in routes where the radio connectivity of VANET is more probable on them. Therefore, it is provisioned to estimate the radio connectivity of each road segment and cache them locally in the VANET-Connectivity table. To do this, the Neighboring table is used. In addition, the received information of other nodes are used for updating the information of this table. When a node receives an updated value for each road segment, the old data is replaced by this new value for that entry in the VANET-Connectivity table.

Radio connectivity calculation by using local neighboring information is done as the following: considering there are $n$ vehicles in node's local Neighboring table, it can be easily seen that that particular road segment is divided into $n+2$ consecutive semi-parts. Therefore, the connectivity probability of that road segment can be calculated by (4):

$$
P_{\text {Connectiviy }}=\left(\sum_{i=1}^{n+2} l_{i}\right) / L
$$

where PConnectivity is radio connectivity probability of the road segment, $\mathrm{L}$ is the road segment length $(m)$ and li are the length $(m)$ of those road segment semi-parts that have lengths less than the maximum radio range of the transmitter equipments(e.g. 250 meters).

For calculating the whole path connectivity probability, a procedure similar to Dijkstra is used to find the most reliable route for packets.

\section{F. System Activity}

Each node implements Dijkstra algorithm to find the path with minimum travel time from current location to the destination using its local Travel Time table. This action is repeated when a node is reaching to a junction during its trip. A path that has the least travel time from vehicle current location to the destination is suggested to the driver. After planning the best path, if the cached information of any planned road segments in Travel-time table is older than a predefined threshold, the algorithm releases a Lookup packet for those road segments. The system uses VANET-Connectivity table to find the most radio-connected routes toward the desired road segments, and puts this path into the Lookup packet and broadcasts it. Each node that receives the Lookup packet answers it by a Reply packet if there is any updated value for asked road segments in its local Travel-time table. The Lookup packet is re-broadcasted if the receiver node's road segment is in the planned packet path. Otherwise the lookup packet is dropped. Therefore, the geographical path of packet journey is limited to the most probable radio-connected route that is planned by the proposed algorithm and it can prevent lots of unnecessary broadcastings in the VANET layer. In addition, every node that has the requested data will answer it by a Reply packet and stops rebroadcasting of that lookup packet. Therefore, the limited bandwidth of VANET is not saturated by useless forwarding.

\section{System Evaluation AND Simulation Results}

The proposed algorithm was evaluated by simulation using three simulators as followed:

- OMNET++ [20] to simulate network and exchanged packets between nodes

- SUMO [21] to simulate vehicles movement on digital maps

- VEINS [22], [23] to establish continuous connection and data exchanging between two above simulators.

Geographic area in this study was a $2000 \mathrm{~m} \times 2000 \mathrm{~m}$ with 200 to 1000 moving vehicles. IEEE 802.11p standard has being implemented for radio transceivers.

In this section we will present the performance of the algorithm in two parts. Fig. 1-Fig. 2 show the performance of the system in reducing the travel time and Fig. 3- Fig. 4 show how this algorithm can optimize VANET bandwidth usage.

Fig. 1 presents the system efficiency for reducing travel time, when different percentages of participating vehicles are equipped with the proposed system. The percentage of 
equipped nodes to the total number of participating vehicles is called Penetration. This figure shows that in all densities, increasing the penetration will lead to a better performance of the system and the improvement of $30 \%$ has been reached. By increasing the density of total moving vehicles, the algorithm has a higher performance, because the VANET connectivity is better and traffic information can be exchanged more efficient.

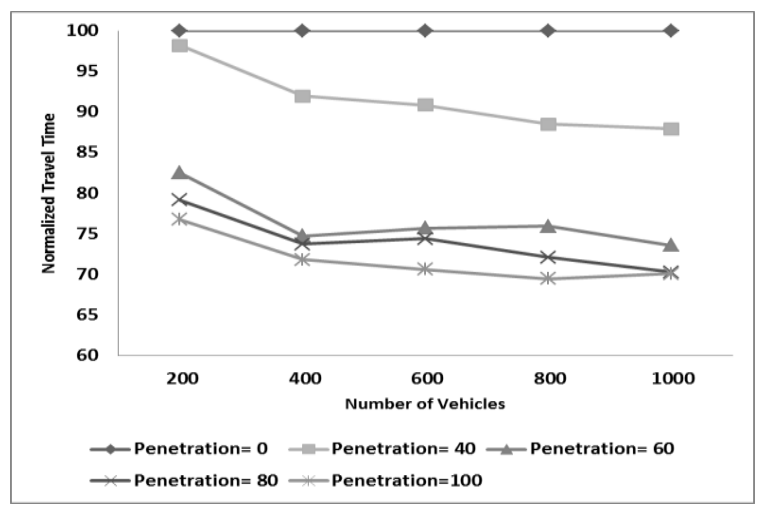

Fig. 1. System efficiency.

Fig. 2 shows the effect of caching on the system accuracy. As this figure presents, in all penetrations increasing the cache time not only does not cause drawbacks in system performance in reducing travel time, but also in high penetrations, it has a good influence in improving the algorithm efficiency. This is because that by caching the information in local tables, the need to send Lookup packets are reduced and the collision probability of packets is decreased. The more cache time will leads to the less lookup need and this leads to less collision probability. Therefore, traffic information can be exchanged more successful and the system can work by a higher performance.

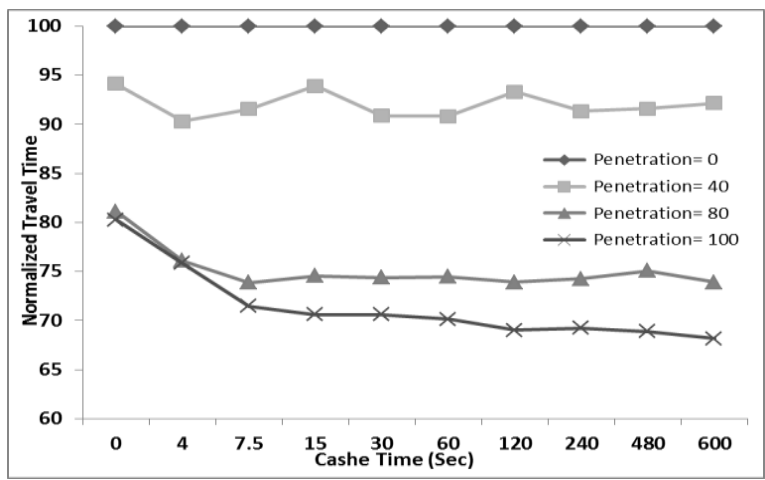

Fig. 2. Effect of cache time on system accuracy.

Fig. 3 shows the effect of adaptive broadcasting for Normal packets and Super-peer packets. The graph presents the percentage of total sent packets when adaptive broadcasting is implemented to the total sent packets when fixed periods are used. The graph shows that by implementing the adaptive broadcasting, system sent only between 20 to 26 percent comparing to send packets in fixed mode in similar conditions. It means that by using adaptive broadcasting, the bandwidth of VANET is saved by 74 to 80 percent. This figure also proves that this bandwidth saving is more in high cache times.

Fig. 4 presents the effect of cache time in reducing VANET bandwidth usage. As use of caching will affect the number of Lookup and Reply packets, this figure shows how the percentage of sending Lookup and Reply packets is reduced by using cache time. This figure shows that with implementing a medium cache time (around one minute), the used bandwidth of VANET decreased by $75 \%$. Increasing the cache time to a long time(around 10 minutes) shows the bandwidth usage reduction of $80 \%$. This figure also shows that in high penetrations, increasing the cache time is more effective than low penetrations.

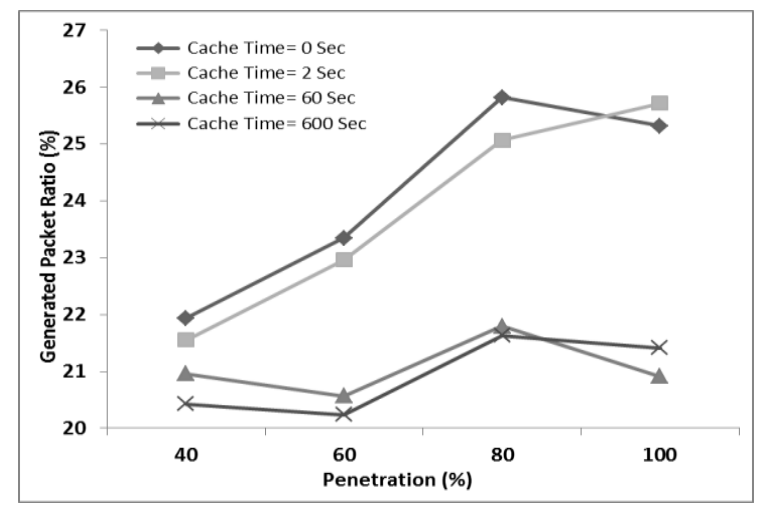

Fig. 3. Effect of adaptive broadcasting on bandwidth usage

Fig. 2-Fig.4 together prove this fact that caching not only has no drawback to the system performance, but it can save the bandwidth of VANET while it preserves the system efficiency in reducing travel time.

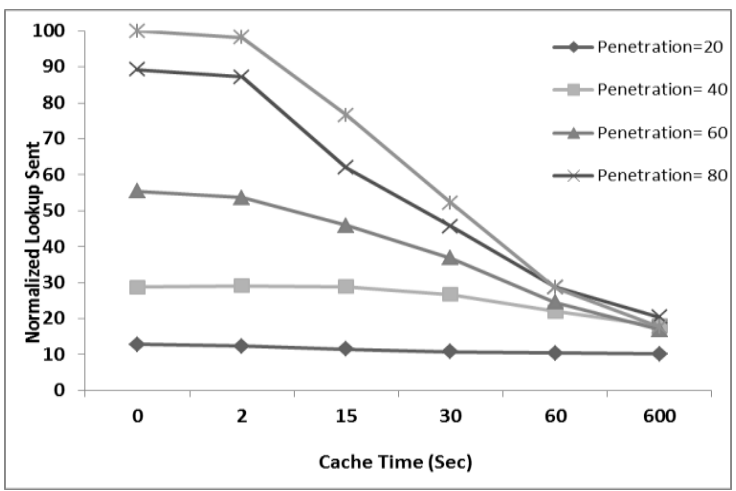

Fig. 4. Bandwidth dependency to cache time.

\section{CONCLUSION AND FUTURE WORK}

By using the proposed algorithm, travel time of vehicles in city roads will be minimized. This system can save the VANET bandwidth by implementing an algorithm that using some techniques such as sending packets from more reliable routes, caching traffic information in local tables and using an adaptive broadcasting approach.

This system can act as an navigation system that reduces travel time for moving vehicles during city trips, while at the other hand, can overcome the limitation of VANET bandwidth problem.

For future work, we will try to evaluate the system sensitivity to the sudden events like an accident. Improving the convergence of information for long distance peers is our later research plans.

\section{REFERENCES}

[1] A. A. Valibak, "Reducing road congestion with a P2P VANET two-purpose routing algorithm for vehicle paths and traffic information 
routes," M.S. Thesis, Language: Persian, Dept. Computer Engineering (E-Learning), Iran University of Science and Technology (IUST), Tehran, Iran, 2013

[2] A. A. Valibak, M. Fathy, and N. M. Balouchzahi, "Reducing time trip with a two-purpose peer-to-peer VANET algorithm for vehicles and traffic information packet routing," presented at the 13'th International Conference on Traffic and Transportation Engineering, Tehran, Iran, Feb. 25-26, 2014

[3] I. Catling and F. O. D. Beek, "SOCRATES: system of cellular radio for traffic efficiency and safety," in Proc. the Vehicle Navigation and Information Systems Conference, vol. 2, pp. 147-150, 1991.

[4] C. Sommer, A. Schmidt, Y. Chen, R. German, W. Koch, and F. Dressler, "On the feasibility of UMTS-based traffic information systems," Ad Hoc Networks, vol. 8, no. 5, pp. 506-517, 2010.

[5] K. Collins and G. M. Muntean, "Route-based vehicular traffic management for wireless access in vehicular environments," in Proc. the. Conference on 68th Vehicular Technology, 2008, pp. 1-5.

[6] M. Seredynski and P. Bouvry, "A survey of vehicular-based cooperative traffic information systems," in Proc. the. 14th International IEEE Conference Intelligent Transportation Systems (ITSC), 2011, pp. 163-168

[7] S. Tsao and C. M. Cheng, "Design and evaluation of a two-tier peer-to-peer traffic information system," Communications Magazine, vol. 49 , no. 5 , pp. $165-172,2011$.

[8] L. Wischoff, A. Ebner, H. Rohling, and M. Lott, " A self-organizing traffic information system," in Proc. the. 57th IEEE Semiannual Vehicular Technology, vol. 4, pp. 2442-2446, 2003.

[9] T. Nadeem, S. Dashtinezhad, C. Liao, and L. Iftode, "TrafficView: Traffic data dissemination using car-to-car communication," ACM SIGMOBILE Mobile Computing and Communications Review, vol. 8, no. 3, pp. 6-9, 2004

[10] S. Goel, T. Imielinski, and K. Ozbay, "Ascertaining viability o based vehicle-to-vehicle network for traffic information dissemination," in Proc. the 7th International IEEE Conference on Intelligent Transportation Systems (ITSC 2004), 2004, pp. 1086 - 1091.

[11] S. Dornbush and A. Joshi, "Street smart Traffic: Discovering and disseminating automobile congestion using VANETs," in Proc. the 65th Vehicular Technology Conference, 2007, pp. 11-15.

[12] M. Jerbi, S. M. Senouci, T. Rasheed, and Y. G. Doudane, "An infrastructure-free traffic information system for vehicular networks," in Proc. the IEEE 66th Vehicular Technology Conference (VTC-2007 Fall), pp. 2007, $2086-2090$.

[13] T. Zhong, B. Xu, P. Szczurek, and O. Wolfson, "Trafficinfo: An algorithm for vanet dissemination of real-time traffic information," in Proc. the 15th World Congress on Intelligent Transport Systems and ITS America's 2008 Annual Meeting, vol. 2, 2008.

[14] A. Lakas and M. Shaqfa, "Geocache: Sharing and exchanging road traffic information using peer-to-peer vehicular communication," in Proc. the Vehicular Technology Conference (VTC Spring), 2011, pp. 1-7.

[15] K. C. Lee, M. Lee, J. Harri, and M. Gerla, "LOUVRE: Landmark overlays for urban vehicular routing environments," in Proc. the 68th Vehicular Technology Conference, 2008, pp. 1-5.

[16] J. Rybicki, B. Pesch, and M. Mauve, "Supporting cooperative traffic information systems through street-graph-based peer-to-Peer networks," in Proc. the 17th GI/ITG Conference on Communication in Distributed Systems, 2011, pp. 121-132.

[17] J. Rybicki, B. Scheuermann, M. Koegel, and M. Mauve, "PeerTIS: a peer-to-peer traffic information system," in Proc. the 6th ACM International Workshop on Vehicular Internetworking, 2009, pp. 23-32.

[18] K. Lu, C. Cheng, and S. Tsao, "An adaptive routing algorithm for two-tier traffic information system," in Proc. the Consumer Communications and Networking Conference (CCNC), 2012, pp. 311-315.

[19] Y. Yang, C. M. Cheng, P. Y. Lin, and S. Tsao, "A real-time road traffic information system based on a peer-to-peer approach," Computers and Communications, pp. 513-518, 2008.

[20] OMNET. OMNET++ Network Simulation Framework. [Online]. Available: http://www.omnetpp.org/.

[21] Simulation of Urban MObility. [Online]. Available: http://www. sumo-sim.org/.

[22] C. Sommer, R. German, F. Dressler, "Bidirectionally Coupled Network and Road Traffic Simulation for Improved IVC Analysis," IEEE Transactions on Mobile Computing, vol. 10, no. 1, pp. 3-15, January 2011.

[23] VEINS. [Online]. Available: http://www. veins.car2x.org/

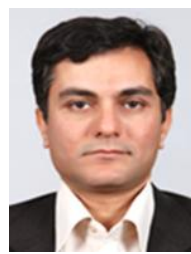

Ali Asghar Valibak received his B.S. degree in electronic engineering from Isfahan University of Technology (IUT), Isfahan, Iran, in 1998 and he received his M.S. degree in information technology (IT) engineering from Iran University of Science and Technology, Tehran, Iran, in 2013. Since 2000 he has worked in mobile telecommunications as a GSM and UMTS/WCDMA planner and optimizer. Currently, he is a member of Vehicular Networks Research Team in Computer Engineering Department at Iran University of Science and Technology under supervision of Dr. Mahmood Fathy and Dr. Kaamran Raahemifar. His main research interests include computer networks, mobile cellular networks and vehicular ad-hoc networks

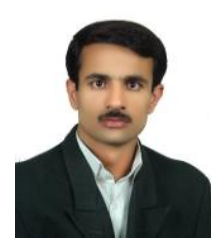

Nik Mohammad Balouchzahi received his B.S. degree in computer engineering from Yazd University, Iran, in 2001 and He received his M.S. degree in computer engineering from Iran University of Science and Technology, Tehran, Iran, in 2004. Since 2004 he has been a faculty member of Electrical and Computer Engineering Department at University of Sistan and Baluchestan. Currently, he is a PhD candidate in computer engineering at Iran University of Science and Technology under supervision of Dr. Mahmood Fathy and Dr. Ahmad Akbari. His main research interests include computer networks, vehicular communications and intelligent transportation systems.

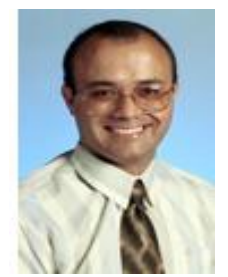

Kaamran Raahemifar received his B.Sc. degree in electrical engineering from Sharif University of Technology, Tehran, Iran in 1985-1998, he received his MASc. degree in electrical and computer engineering Department from Waterloo University, Waterloo, Ontario, Canada in 1991-1993 and he received his $\mathrm{Ph} . \mathrm{D}$. degree from Windsor University, Ontario, Canada in 1996-1999. He was the chief scientist in electronic workbench, Toronto, Ontario, Canada in1999-2000. He joined Ryerson University in Sept. 1999 and was tenured in 2001. Since 2011, he has been a professor with the Department of Electrical and Computer Engineering, Ryerson University. His research interests include optimization in engineering, theory and application, grid optimization and net-zero communities, biomedical signal processing, big data analysis, modelling, simulation, design, and testing, time-based operational circuit designs

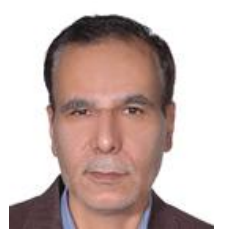

Mahmood Fathy received his B.S. degree in electronics from Iran University of Science and Technology, Tehran, Iran, in 1984, He received his M.S. degree in computer architecture from Bradford University, West Yorkshire, U.K., in 1987, and He received his $\mathrm{Ph}$. D. degree in image processing and its architecture from the University of Manchester Institute of Science and Technology, Manchester, U. K, in 1991. Since 1991, he has been an academic member with the Department of Computer Engineering, Iran University of Science and Technology. His research interests include the quality of service in computer networks, including video and image transmission over Internet, the applications of vehicular ad hoc networks in intelligent transportation systems, real-time image processing, with particular interest in traffic engineering and remote health monitoring.

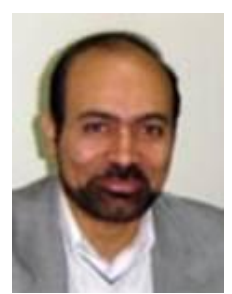

Ahmad Akbari received his BSc. degree in electronics engineering and the MSc. degree in communications engineering from Isfahan University of technology (IUT) in 1986 and 1989 respectively. He received his DEA and Ph.D. degrees in signal processing and telecommunications from university of Rennes 1, Rennes, France in 1992 and 1995 respectively.

In 1996 he joined the Computer Engineering Department at Iran University of Science and Technology (IUST) as an assistant professor, where he is now the director of computer engineering department. His research interests include computer networking, network security, acoustic modeling of speech, robust speech recognition, speech enhancement, implementation of signal processing algorithms, voice applications and interfaces and web technologies. 